\title{
Characterization of plasma polymerized HMDSN films deposited by atmospheric plasma jet
}

\author{
F.V.P. Kodaira a,*, A.H. Ricci Castro ${ }^{\text {a }}$, V. Prysiazhnyi ${ }^{\text {a }}$, R.P. Mota ${ }^{a}$, A. Quade ${ }^{\text {b }}$, K.G. Kostov ${ }^{\text {a }}$ \\ a UNESP, São Paulo State University, Av. Dr. Ariberto Pereira da Cunha, 333 Guaratinguetá, SP, Brazil \\ ${ }^{\mathrm{b}}$ INP, Leibniz-Institute for Plasma Science and Technology, Felix-Hausdorff Str. 2, 17489 Greifswald, Germany
}

\section{A R T I C L E I N F O}

\section{Article history:}

Received 21 March 2016

Revised 18 October 2016

Accepted in revised form 30 November 2016

Available online 2 December 2016

\section{Keywords:}

Plasma polymer

HMDSN

Atmospheric pressure plasma jet

APPJ

Polymeric films

\begin{abstract}
A B S T R A C T
Deposition of plasma polymers using atmospheric pressure plasma jet in mixture of argon, air and hexamethyldisilazane (HMDSN) was studied. The HMDSN monomer, used as film precursor, was carried to the jet nozzle by an auxiliary airflow and mixed with principal argon flow. The films were deposited for different times using mean discharge power of $2.8 \mathrm{~W}$, main argon flow of 1 SLM while the carrier flow (air + HMDSN) was 0.1 SLM. The water contact angles measured across the obtained HMDSN plasma polymer film showed two welldefined areas, one hydrophilic, with contact angle near zero degrees and one hydrophobic, with contact angle around 100 degrees. The FTIR spectra showed that part of the original structure of HMDSN monomer was preserved and new chemical bonds were observed. XPS analysis showed that the deposited coatings are inorganic $\mathrm{SiO}_{2}$-like coatings. The films thickness was evaluated and it reached values about $2 \mu \mathrm{m}$ in the center and decreased to the border (in continuous deposition mode more rapidly than in the pulsed one). Roughness values were obtained and it is shown that they varied for each region of the film.
\end{abstract}

(C) 2016 Published by Elsevier B.V.

\section{Introduction}

Plasma polymers have attracted much attention due to their growing applications in different areas such as medical, biological, electrical, mechanical, coatings and others [1-3]. The plasma polymers have different characteristics in comparison with the regular polymers, because the plasma polymerization process is unlike any chemical process. It has plenty of advantages, such as absence or small quantities of chemical wastes and high control of the deposited films. The films have a highly cross-linked structure, well-defined thickness from nm to $\mu \mathrm{m}$ scale, pinhole free, homogeneous and insoluble in mild acid or basic medium [1,4-6].

Low-pressure systems have been commonly used for plasma polymerization for many years. However, atmospheric pressure systems are now being studied for this purpose due to some advantages over the low-pressure systems. Atmospheric pressure plasmas are attractive due to lack of expensive vacuum systems, which also requires long time to evacuate the deposition chamber. One of most studied devices used to produce atmospheric pressure plasma is the Dielectric Barrier Discharge (DBD). More recently, the plasma jet has been introduced as an alternative to the DBD for atmospheric pressure plasma polymerization [7-12].

The free energetic electrons in the plasma break the monomer into ions, excited species, more free energetic electrons, and mainly free

\footnotetext{
* Corresponding author.

E-mail address: kodaira.felipe@gmail.com (F.V.P. Kodaira).
}

radicals [1,5]. Plasma polymerization is a process where polymers are grown by successive addition of free radicals, which are generated from a starting monomer broken by plasma.

The hexamethyldisilazane (HMDSN) is known to produce silicon and carbon based plasma polymers, used in different applications, such as in microelectronics for protection coating, optics, sensors and solar cells [13-17]. The low-pressure plasma polymerized HMDSN film is well studied and it is known to have hydrophobic character and deposition rate ranging from 1 to $110 \mathrm{~nm} / \mathrm{min}$ depending on the applied power $[13,18,19]$. For deposition using atmospheric pressure plasma jet or atmospheric pressure DBD, the HMDSN films shows deposition rates ranging from 10 to $30 \mathrm{~nm} / \mathrm{min}$ [12,20,21]. In this work an atmospheric pressure plasma jet was used to polymerize HMDSN films. Electrical characterization of the plasma jet was done by acquiring current and voltage waveforms and measuring the dissipated power. The films properties (including along its diameter as the deposition was not homogeneous) studied in this work were the wettability (analyzing the value of the water contact angle), chemical structure by ATR-FTIR and XPS, and thickness/roughness analysis by confocal microscopy.

\section{Experimental setup}

A scheme of the plasma jet is shown in Fig. 1. The device consists of a $3 \mathrm{~mm}$ inner diameter glass tube and a $1 \mathrm{~mm}$ thick copper wire electrode centered inside it. This electrode is connected to a high voltage power 


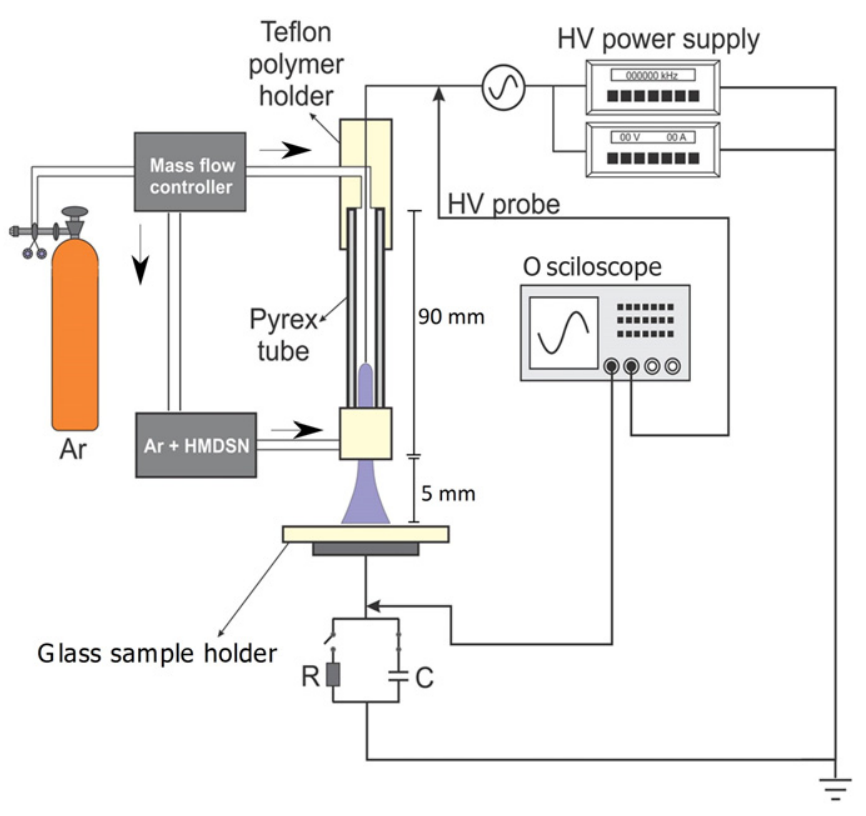

Fig. 1. Scheme of the experimental setup for the deposition of films from the HMDSN monomer.

source (Minipuls 4, GBS Elektronik, Germany). The high voltage amplitude was $12 \mathrm{kVp}$-p, while the applied frequency was $19 \mathrm{kHz}$. Due to a specific coupling between the plasma jet and the power supply, one period of the voltage signal contained two semi-cycles with higher and lower amplitude (the shape is presented in Fig. 2), which was described in details elsewhere [22].

Below the plasma jet it is located a $4 \mathrm{~mm}$ thick soda-lime glass sample holder placed on a metal plate grounded electrode. The system has two gas inlets: main argon flow that feeds the jet from the upper side and another flow that carries the HMDSN monomer to the tip of the jet (called here air + HMDSN flow). The secondary gas inlet located close to the nozzle was introduced to prevent polymerization on the HV electrode. Electrical characterization of the APPJ was done by measuring the voltage and current (through a $100 \mathrm{Ohm}$ inductance-free resistor connected in series) waveforms, and voltage on external capacitor $(10 \mathrm{nF})$ when the dissipated power was calculated using Lissajous figures. The data were acquired via HV probe (P6015A, Tektronix, USA),

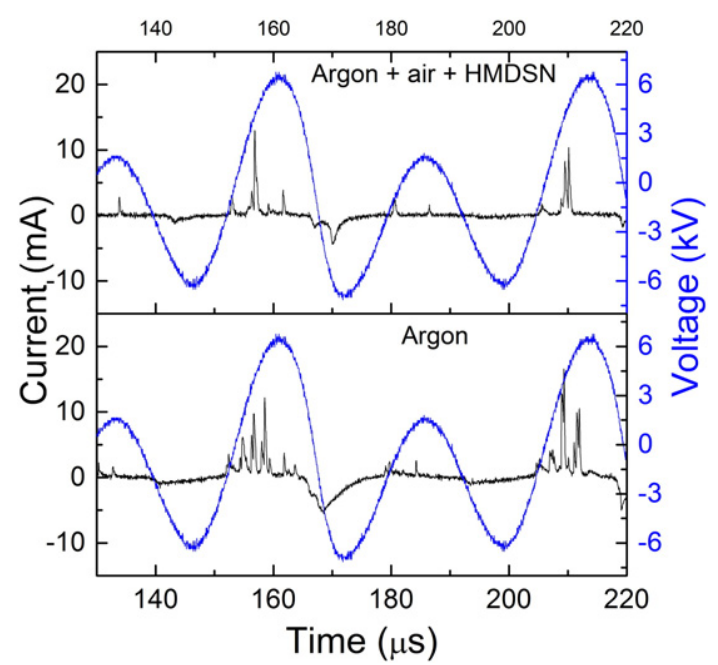

Fig. 2. Applied voltage and discharge current waveforms in a time interval for argon + HMDSN flow (top) and for argon only (bottom). low-voltage probe (P2220, Tektronix, USA) and collected using digital oscilloscope (TDS 3032B $300 \mathrm{MHz}$, Tektronix, USA).

The samples were placed on the glass sample holder on a fixed distance of $5 \mathrm{~mm}$ far from the tip of the jet. This distance was found experimentally and was chosen to obtain better films with less powder. Two different regime of operation were used to deposit the films, "continuous" and "pulsed". When operated in the continuous regime, the main argon flow and the air + HMDSN flow were turned on during the entire deposition time. In the pulsed mode, the main argon flux remained "on", while the air + HMDSN was switched "on" for $10 \mathrm{~s}$ with $5 \mathrm{~s}$ turned "off". In pulsed mode only the time when the air + HMDSN flux is switched "on" was taken into consideration so that deposition time in both modes had the same deposition time. The main argon flux was fixed at 1 SLM and the air + HMDSN flux at 0.10 SLM.

Measurements of contact angle were carried out on a goniometer ( $300 \mathrm{~F} 1$, Rame Hart, USA). Water drops of $1 \mu \mathrm{L}$ were placed along the center of the sample at distances of $1 \mathrm{~mm}$ from each other in order to make a radial scan of the film wettability. $5 \mathrm{~cm} \times 2 \mathrm{~cm}$ glass plates were used as substrates.

The FTIR spectra were obtained using a Spectrum 100 spectrometer (Perkin Elmer, USA) in the range of $4000 \mathrm{~cm}^{-1}$ to $650 \mathrm{~cm}^{-1}$. The measurements were performed in the center of the film. Polyethylene substrates were used for infrared spectroscopy, because its absorption peaks in the infrared region are not coincident with the ones from the polymeric film.

Surface chemical analysis was done using x-ray photoelectron spectroscopy (Axis Ultra, Kratos, UK) equipped with monochromatic Al Ka (1486.6 eV) source with charge neutralization. All measurements were done with $150 \mathrm{~W}$ power from the $\mathrm{x}$-ray source (15 kV voltage and $10 \mathrm{~mA}$ current), like the FTIR analysis, the measurements were done in the center of the samples. Survey spectra were measured from $250 \mu \mathrm{m}$ spot size, while high-resolution scans were obtained from $350 \mu \mathrm{m}$ spot with $80 \mathrm{eV}$ pass energy for both cases. Spectra processing were done in CasaXPS software.

The thickness and roughness values were evaluated by a confocal microscope (DCM 3D, Leica, Germany). A scalpel blade was used to scratch the film on the substrate and this indentation was used to obtain the thickness. The thickness measurements were taken every $1.5 \mathrm{~mm}$ starting from the center of the film in order to obtain the thickness distribution. Roughness values were acquired from different regions of the film.

\section{Results and discussion}

The APPJ system operated in Ar only showed a typical multifilament behavior [23]. Adding flow of air and HMDSN led to decrease of plasma plume light intensity, and made plasma colored slightly more pink. However, emission spectra did not show presence of new emission lines, rather a ratio between argon lines intensity and nitrogen bands intensity decreased. Modeling of spectra using SpecAir software (of nitrogen band $0-2$ at $380 \mathrm{~nm}$ ) showed that adding flows of air and HMDSN did not change the gas temperature that was estimated to be $800 \pm$ $200 \mathrm{~K}$.

The electrical characterization of plasma jet is presented in the Fig. 2 . The upper graph shows the current and voltage signal when both main argon flow and the auxiliary air flow mixed with HMDSN are "on", while the lower one shows the signals for the APPJ operated with the main argon flow only. The voltage signal is the same in both figures, but the current signal is different in each case. For argon flow only plasma jet current exhibits more discharge peaks than for the case of $\mathrm{Ar}+$ air + HMDSN jet. Inserting monomer into the Ar flow changes the conditions of the discharge by increasing the energy losses of electrons in collisions $[24,25]$. The power of the jet was obtained by the Lissajous figure method [8]. The jet with only Ar power was $4.3 \mathrm{~W}$ while adding air + HMDSN to main flow leads to decrease of the dissipated power to $2.8 \mathrm{~W}$. 


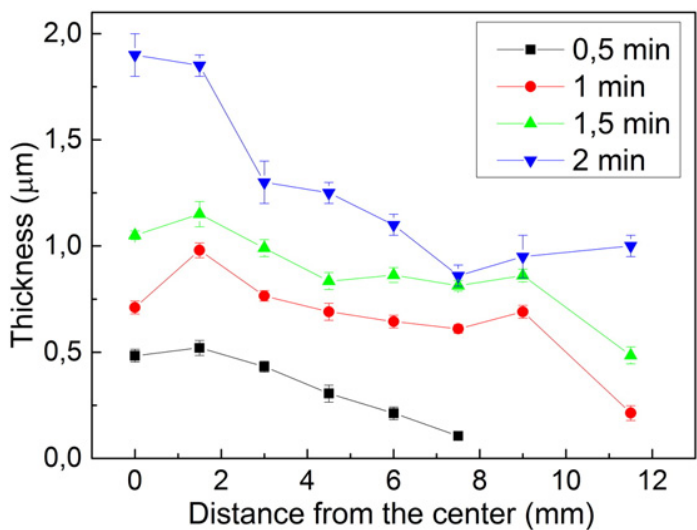

Fig. 3. Mapping of the thickness of the films measured from the center to the border. The films were deposited for different times in the pulsed mode.

Values of the films thickness produced using the pulsed mode are presented in Fig. 3. The thickness of the film decreased radially, while the decrease of film thickness for continuous mode was higher than for the pulsed mode. The radicals are carried outwards by the gas flux and diffusion so that their probability of reacting further distances of the substrate decreases. Other species like ions and electrons have shorter lifetime, so their chances to reach further distances are even lower. The pulsed mode seems to homogenize the film thickness as it was observed for the wettability. For example, for $1.5 \mathrm{~min}$ film deposition, about $1 \mu \mathrm{m}$ film was deposited for both deposition modes. However, $9 \mathrm{~mm}$ away from the center for the continuous mode the film thickness was about $0.5 \mu \mathrm{m}$ thick, while for the pulsed one it was still near $0.9 \mu \mathrm{m}$. The film thickness dropped to about $0.5 \mu \mathrm{m}$ for the pulsed mode on the distance of $12 \mathrm{~mm}$ from the center. Taking into account both deposition modes, and data for $0.5 \mathrm{~min}, 1 \mathrm{~min}, 1.5 \mathrm{~min}$ and 2 min deposition times, an average deposition speed of about $1 \mu \mathrm{m} /$ min was achieved.

The deposited HMDSN films were not homogeneous, since the plasma plume is directed on a single point, and the film properties change with the distance from the plume. Fig. 4 shows films deposited on glass substrates, on the right hand side one can see films in the way they look just after the polymerization for the pulsed mode. The radius of the external halo is about $1 \mathrm{~cm}$. The plasma plume spreads radially over the substrate, covering about the same area. On the left hand side of Fig. 4 are shown the films after rinsing in ultrasonic bath for $1 \mathrm{~min}$.

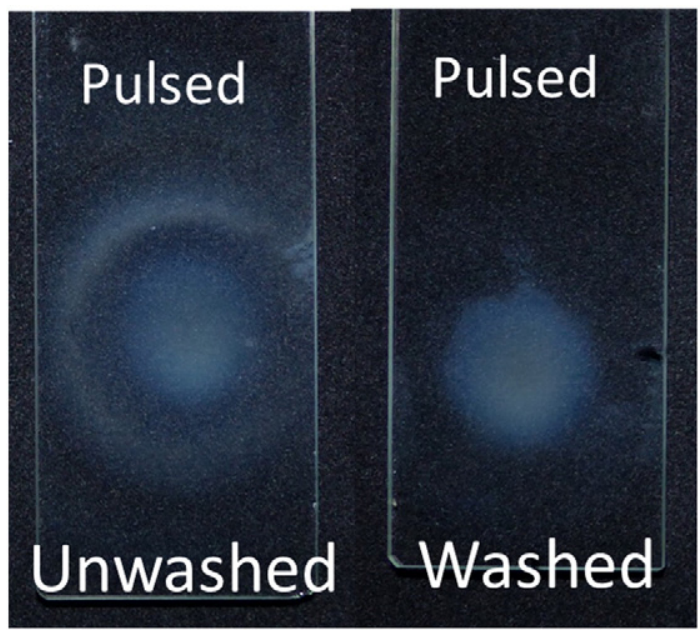

Fig. 4. Photo of the deposited films using the pulsed mode before (right) and after (left) washing in water in ultrasonic bath for $1 \mathrm{~min}$. Deposition time $2 \mathrm{~min}$.

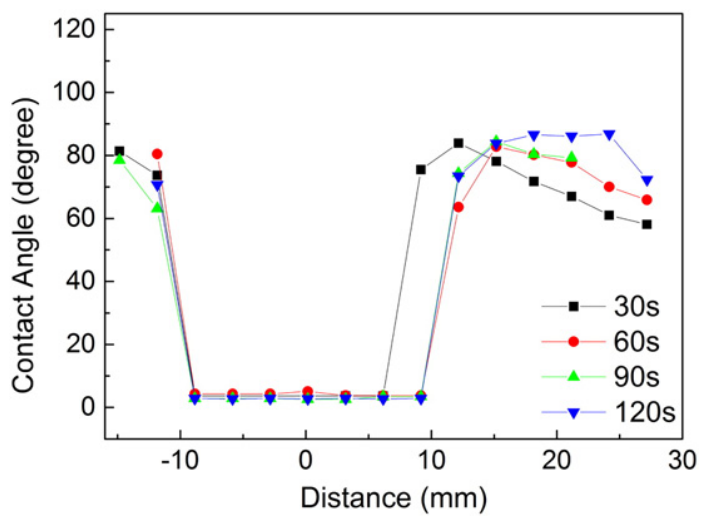

Fig. 5a. Diametric mapping of the water contact angle for films deposited for different times in pulsed mode.

It can be seen that the central part of the film is visually still attached to the surface while the external halo seems to be washed away. This halo is weakly bonded to the substrate and it may be formed by oligomers detached from the central region and carried out by the gas flow of the jet.

In Figs. $5 a$ and $5 b$ show the distribution of the water contact angle for unwashed and washed samples for pulsed deposition mode, respectively. It can be seen that the film's central part was not removed by rinsing the samples in water. WCA value dependence has a clear edge at the border of the halo region for both pulsed and continuous deposition modes also. The pulsed mode led to slightly wider and a more homogeneous film in the point of view of wettability. All conditions resulted in hydrophilic films, with contact angle near zero for the pulsed mode and below 30 degrees for the continuous mode, in the region under the plasma plume. The radius of hydrophilic region was about $10 \mathrm{~mm}$ for all cases. A hydrophobic characteristic, with WCA values about 100 degrees for both pulsed and continuous modes, was observed in the regions more than $10 \mathrm{~mm}$ away from the center of the plasma plume. Polymers treated by argon plasma jet present a similar wettability behavior $[8,26$, 27], being hydrophilic in the region near the plume. On the other hand, HMDSN films grown by plasma polymerization at low pressure feature values for the water contact angle around $100^{\circ}[18]$. Thus, the region outside the hydrophilic area shows values compatible to the ones for HMDSN films. The contact angle of untreated glass substrates is about $50^{\circ}$, as shown in those graphs.

Almost no variation of the contact angle values with the time were observed in the hydrophilic region of the films deposited using the pulsed mode (i.e. exposition to argon plasma jet for $5 \mathrm{~s}$ after every

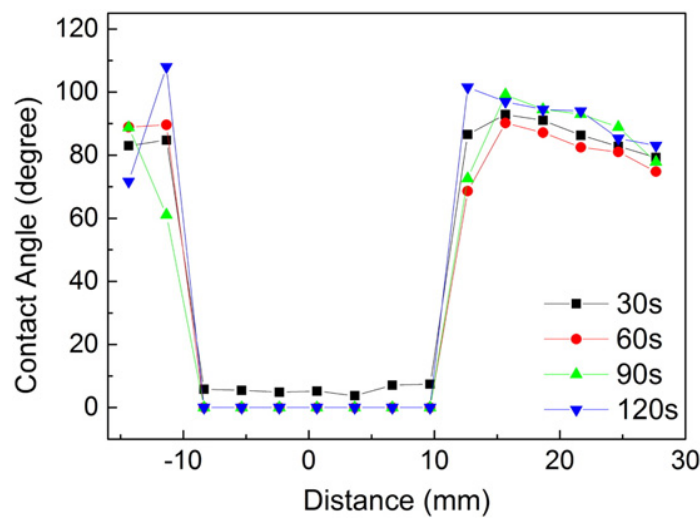

Fig. 5b. Diametric mapping of the water contact angle for films deposited for different times in pulsed mode after washing in water in ultrasonic bath for $1 \mathrm{~min}$. 


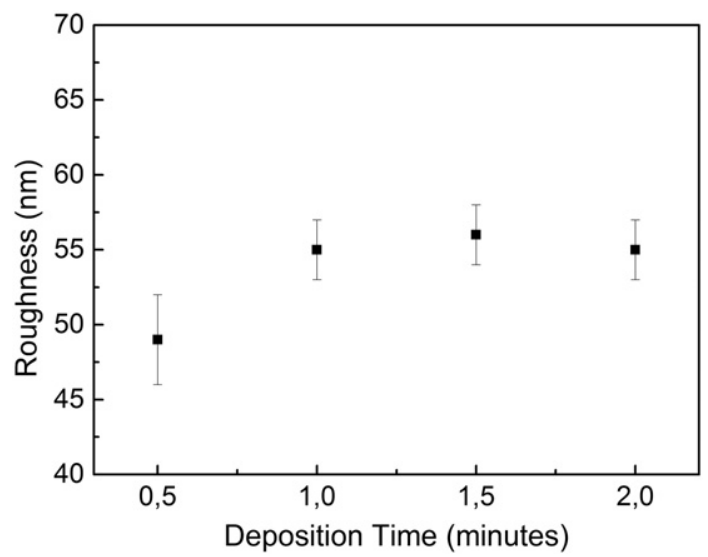

Fig. 6. Roughness of the films deposited for different times using the pulsed mode. The measures were taken in the film center.

$10 \mathrm{~s}$ of deposition). However, on the hydrophobic region, the jet operation mode seems to have little effect on deposited films since both pulsed and continuous mode showed similar results.

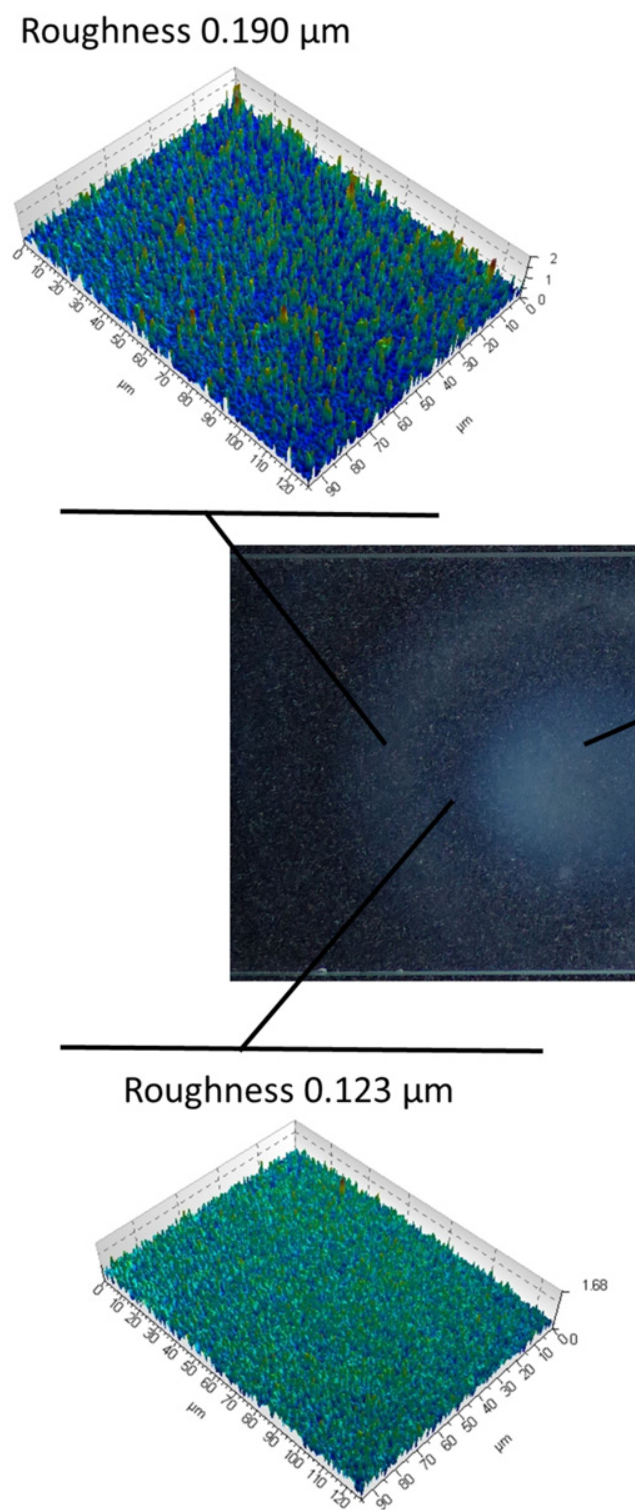

Films deposited in continuous mode had higher roughness than the ones obtained in pulsed mode. The roughness values for the film deposited using the pulsed mode is shown in Fig. 6. Both modes also exhibited roughness saturation for deposition times over $1 \mathrm{~min}$. All those roughness values were measured in the center of the film.

The deposited films were visually non-homogeneous. One can observe an inner circle, followed by a transparent ring and then an outside halo. The roughness was not the same in all these regions. The Fig. 7 shows the roughness and topography for each of the regions. One can observe that the film is smoother in the inner circle and far outside the halo, the roughness increases in the transparent circle and even more in the outside halo. As explained for Fig. 4, oligomers may have been detached from the center region by the plasma and dragged radially by the gas flow to this external region, making it rough.

Chemical analyses of the deposited films were done using FTIR and XPS techniques. It is important to note that both techniques showed that samples deposited using both pulsed and continuous deposition had same chemical structure. Fig. 8 shows IR spectra of polyethylene substrate (a) and plasma deposited film in pulsed mode on PE (b). It was decided to present spectra deposited on soft $0.2 \mathrm{~mm}$ thick PE substrate, as glass have wide peaks below $1200 \mathrm{~cm}^{-1}$, overlapping with the peaks, thus making peak identification rather complicated task.

$$
\text { Roughness } 0.069 \mu \mathrm{m}
$$

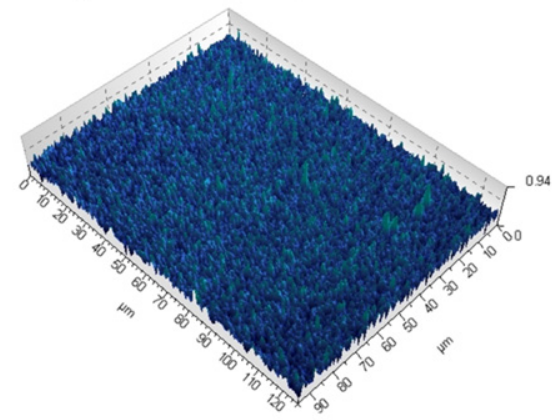

Fig. 7. Roughness and topography of the different regions observed in the deposited films using the pulsed mode. 


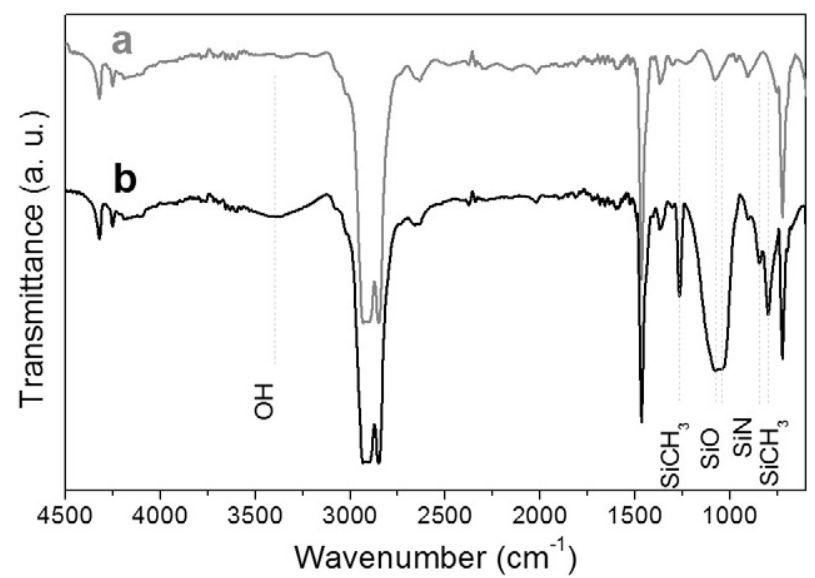

Fig. 8. FTIR spectra of the films deposited for different times using the continuous and pulsed modes.

After comparing IR spectra measured from clean PE substrate and the one with deposited film, six distinct peaks were assigned to be from the deposited film. They are wide $\mathrm{OH}$ band at about $3400 \mathrm{~cm}^{-1}$, peaks of $\mathrm{SiCH}_{3}$ at $800 \mathrm{~cm}^{-1}$ and $1260 \mathrm{~cm}^{-1}$, $\mathrm{SiO}$ peaks at $1040 \mathrm{~cm}^{-1}$ and $1075 \mathrm{~cm}^{-1}$, and low-amplitude SiN peak at $845 \mathrm{~cm}^{-1}[19,20]$. Typically observed four peaks of $\mathrm{CH}_{2} / \mathrm{CH}_{3}$ at about $2900 \mathrm{~cm}^{-1}$ were overlapped by the PE substrate, but were observed on the films deposited on glass substrate.

The difference between continuous and pulsed mode is in the ratio between the observed peaks. Table 1 shows normalized (to SiN as 1 ) intensity of peaks for both deposition modes.

To perform extensive surface chemical analysis, X-ray photoelectron spectroscopy was done on a set of samples: continuous deposition unwashed and washed, pulsed deposition unwashed and washed. All samples were prepared with same deposition time of $1 \mathrm{~min}$. The chemical composition of all samples were same, all samples contained carbon $(6.6 \pm 1.0 \%)$, oxygen $(65.1 \pm 0.8 \%)$ and silicon $(28.2 \pm 0.4 \%)$. It was not expected, but there was no nitrogen detected on neither of the samples, though showing correlation between the FTIR and XPS data. The explanation of this fact was given in [20], where authors proposed that $\mathrm{SiN}$ is not stable when exposed to ambient air. This can explain presence of SiN peak obtained from IR spectrum (as it is measured from the whole film, i.e. $\mu \mathrm{m}$ scale), while nitrogen was not found at film surface (around $10 \mathrm{~nm}$ ).

Oxygen to silicon ratio was also almost constant for all samples, showing the value of $2.3 \pm 0.3$, therefore showing that the deposited films were not glass-like. The fitting of the high-resolution Si 2p peak demonstrated a presence of four components [28]. The percentage of $\mathrm{Si}(-\mathrm{O})_{4}$ was exceeding $50 \%$, and the percentage of $\mathrm{Si}(-\mathrm{O})_{3}-35 \%$, allowing us to conclude that the deposited coatings were inorganic $\mathrm{SiO}_{2}$-like coatings. The stability of the fitting components allows us to conclude that films are stable and non-soluble.

\section{Conclusion}

Polymer deposition using the atmospheric pressure plasma jet has been shown to be an alternative process to the conventional lowpressure plasma polymerization, producing films with distinct

Table 1

Ratio of peak intensity for various chemical bonds normalized to SiN peak intensity.

\begin{tabular}{lllllll}
\hline & $\mathrm{OH}$ & $\mathrm{SiCH}_{3}(1260)$ & $\mathrm{SiCH}_{3}(800)$ & $\mathrm{SiN}$ & $\mathrm{SiO}(1075)$ & $\mathrm{SiO}(1040)$ \\
\hline Pulsed & 0.3 & 1.5 & 1.8 & 1 & 2.6 & 2.55 \\
Continuous & 0.35 & 1.6 & 1.6 & 1 & 2.4 & 2.55
\end{tabular}

characteristics. The deposited films were not homogeneous, depositing more material in the center, while on the edges the film thickness was decreasing. Two modes of deposition were studied: continuous (when mixture of argon, air and HMDSN was supplied throughout whole deposition time) and pulsed ( $10 \mathrm{~s}$ of $\mathrm{Ar}+$ air + HMDSN followed by $5 \mathrm{~s}$ of $\mathrm{Ar}$ plasma only). As measurements are taken radially outwards from the center of the film, its characteristics change from hydrophilic to hydrophobic, differently from the films obtained by low-pressure plasma polymerization techniques that are sole hydrophobic. The pulsed mode of operation resulted in more homogeneous films compared with continuous mode. The roughness analysis showed that the morphology of the films changes along its radius. Films deposited using the continuous mode (the RMS value in the center was about $67 \mathrm{~nm}$ ) were rougher than using the pulsed one (the corresponding value was about $58 \mathrm{~nm}$ ). The films thickness values decrease from the center to the border of the film regardless of the mode (pulsed or continuous), but the thickness of the films made by continuous mode deposition falls more quickly. The deposition rate of both continuous and pulsed mode were around $1 \mu \mathrm{m} / \mathrm{min}$, which is much higher than the deposition rates observed in previous works being from 1 to $110 \mathrm{~nm} / \mathrm{min}$. The FTIR and XPS analysis showed that the deposited coatings were inorganic $\mathrm{SiO}_{2}$ like coatings with almost no nitrogen in its structure in the central region with $1 \mathrm{~cm}$ radius. Chemical structure of the deposited films was stable to washings in water and the films did not exhibit aging effect in ambient air.

\section{Acknowledgements}

The authors would like to thank CAPES and CNPq for the financial support and Dr. Olena Solomenko for her help with emission spectroscopy measurements.

\section{References}

[1] D.L. Flamm, O. Auciello, R. D'Agostino, Plasma Deposition, Treatment, and Etching of Polymers: The Treatment and Etching of Polymers, Elsevier, 2012.

[2] P. Chu, Plasma-surface modification of biomaterials, Mater. Sci. Eng. R. Rep. 36 (2002) 143-206, http://dx.doi.org/10.1016/S0927-796X(02)00004-9.

[3] A. Brooks, S. Woollard, G. Hennighan, T. von Werne, Plasma polymerization: a versatile and attractive process for conformal coating, Proc. SMTA Int. Conf 2012, pp. 1-6.

[4] D. Thiry, S. Konstantinidis, J. Cornil, R. Snyders, Plasma diagnostics for the lowpressure plasma polymerization process: a critical review, Thin Solid Films 606 (2016) 19-44, http://dx.doi.org/10.1016/j.tsf.2016.02.058.

[5] H.K. Yasuda, Plasma Polymerization, Elsevier Science, 2012https://books.google. com.br/books?id=5Fi1liMCdtsC.

[6] N. Inagaki, Plasma Surface Modification and Plasma Polymerization, CRC Press, 1996.

[7] C. Tendero, C. Tixier, P. Tristant, J. Desmaison, P. Leprince, Atmospheric pressure plasmas: a review, Spectrochim. Acta B At. Spectrosc. 61 (2006) 2-30, http://dx. doi.org/10.1016/j.sab.2005.10.003.

[8] K.G. Kostov, T.M.C. Nishime, A.H.R. Castro, A. Toth, L.R.O. Hein, Surface modification of polymeric materials by cold atmospheric plasma jet, Appl. Surf. Sci. 314 (2014) 367-375, http://dx.doi.org/10.1016/j.apsusc.2014.07.009.

[9] O. Carton, D. Ben Salem, J. Pulpytel, F. Arefi-Khonsari, Improvement of the water stability of plasma polymerized acrylic acid/MBA coatings deposited by atmospheric pressure air plasma jet, Plasma Chem. Plasma Process. 35 (2015) 819-829, http:// dx.doi.org/10.1007/s11090-015-9634-9.

[10] I. Gordeev, M. Šimek, V. Prukner, A. Artemenko, J. Kousal, D. Nikitin, et al., Deposition of poly(ethylene oxide)-like plasma polymers on inner surfaces of cavities by means of atmospheric-pressure SDBD-based jet, Plasma Process. Polym. (2016) n/a, http:// dx.doi.org/10.1002/ppap.201500214.

[11] M.J. Shenton, G.C. Stevens, Surface modification of polymer surfaces: atmospheric plasma versus vacuum plasma treatments, J. Phys. D. Appl. Phys. 34 (2001) 2761-2768, http://dx.doi.org/10.1088/0022-3727/34/18/308.

[12] C. Huang, S.Y. Wu, Y.C. Chang, Synthesis of organosilicon film on polycarbonate by means of low-temperature atmospheric-pressure plasma jet, IEEE Trans. Plasma Sci. 38 (2010) 1101-1105, http://dx.doi.org/10.1109/TPS.2010.2044894.

[13] F. Kraus, S. Cruz, J. Muller, Plasmapolymerized silicon organic thin films from HMDSN for capacitive humidity sensors, Sensors Actuators B Chem. 88 (2003) 300-311, http://dx.doi.org/10.1016/S0925-4005(02)00373-8.

[14] M.L.P. Da Silva, I.H. Tan, A.P. Nascimento Filho, E. Galeazzo, D.P. Jesus, Use of plasma polymerized highly hydrophobic hexamethyldissilazane (HMDS) films for sensor development, Sensors Actuators B Chem. 91 (2003) 362-369, http://dx.doi.org/10. 1016/S0925-4005(03)00112-6. 
[15] H.R. Lee, D.J. Kim, K.H. Lee, Anti-reflective coating for the deep coloring of PET fabrics using an atmospheric pressure plasma technique, Surf. Coat. Technol. 142-144 (2001) 468-473, http://dx.doi.org/10.1016/S0257-8972(01)01137-9.

[16] D. Peters, J. Muller, T. Sperling, Insulation and passivation of three-dimensional substrates by plasma-CVD thin films using silicon-organic compounds, Mater. Sci. Eng. A 139 (1991) 380-384, http://dx.doi.org/10.1016/0921-5093(91)90646-5.

[17] H. Biederman, Plasma Polymer Films, Imperial College Press, 2004https://books.google.com.br/books?id=KgK-qc0wEx0C.

[18] F.V.P. Kodaira, R.P. Mota, P.W.P. Moreira, Thin films growth by PIIID technique from hexamethyldisilazane/argon mixture, Surf. Coat. Technol. (2015)http://dx.doi.org/ 10.1016/j.surfcoat.2015.09.063.

[19] E. Vassallo, A. Cremona, F. Ghezzi, F. Dellera, L. Laguardia, G. Ambrosone, et al., Structural and optical properties of amorphous hydrogenated silicon carbonitride films produced by PECVD, Appl. Surf. Sci. 252 (2006) 7993-8000, http://dx.doi.org/10. 1016/j.apsusc.2005.10.017.

[20] S.H. Yang, C.H. Liu, C.H. Su, H. Chen, Atmospheric-pressure plasma deposition of $\mathrm{SiO}_{\mathrm{x}}$ films for super-hydrophobic application, Thin Solid Films 517 (2009) 5284-5287, http://dx.doi.org/10.1016/j.tsf.2009.03.083.

[21] M. Šíra, D. Trunec, P. St'ahel, V. Buršíková, D. Franta, Deposition of organic polymers at higher substrate temperatures in atmospheric pressure glow discharge, Czechoslov. J. Phys. 56 (2006) 1377-1382, http://dx.doi.org/10.1007/s10582-0060377-y.
[22] V. Prysiazhnyi, A.H.C. Ricci, K.G. Kostov, On power measurements of single-electrode low-power Ar plasma jets, Braz. J. Phys. 1-7 (2016)http://dx.doi.org/10.1007/ s13538-016-0433-4.

[23] J.L. Walsh, F. Iza, N.B. Janson, V.J. Law, M.G. Kong, Three distinct modes in a cold atmospheric pressure plasma jet, J. Phys. D. Appl. Phys. 43 (2010) 075201, http://dx. doi.org/10.1088/0022-3727/43/7/075201.

[24] B. Van Gessel, R. Brandenburg, P. Bruggeman, Electron properties and air mixing in radio frequency driven argon plasma jets at atmospheric pressure, Appl. Phys. Lett. 103 (2013) 3-7, http://dx.doi.org/10.1063/1.4817936.

[25] J.L. Walsh, J.J. Shi, M.G. Kong, Contrasting characteristics of pulsed and sinusoidal cold atmospheric plasma jets, Appl. Phys. Lett. 88 (2006)http://dx.doi.org/10. 1063/1.2198100.

[26] A. Van Deynse, P. Cools, C. Leys, R. Morent, N. De Geyter, Surface modification of polyethylene in an argon atmospheric pressure plasma jet, Surf. Coat. Technol. 276 (2015) 384-390, http://dx.doi.org/10.1016/j.surfcoat.2015.06.041.

[27] T.K. Yun, J.H. Kim, D.K. Lee, Enhancing the surface wettability of poly(ethylene terephthalate) film by atmospheric pressure plasma treatment with $\mathrm{Ar}$ and $\mathrm{N}_{2}$ Gas mixture, Mol. Cryst. Liq. Cryst. 586 (2013) 188-195, http://dx.doi.org/10.1080/ 15421406.2013.853556.

[28] D.S. Wavhal, J. Zhang, M.L. Steen, E.R. Fisher, Investigation of gas phase species and deposition of $\mathrm{SiO}_{2}$ films from $\mathrm{HMDSO} / \mathrm{O}_{2}$ plasmas, Plasma Process. Polym. 3 (2006) 276-287, http://dx.doi.org/10.1002/ppap.200500140. 